

\title{
Coherent soliton pattern formation in a fiber laser
} Adil Haboucha, Hervé Leblond, Mohamed Salhi, Andrey Komarov, François Sanchez

\section{To cite this version:}

Adil Haboucha, Hervé Leblond, Mohamed Salhi, Andrey Komarov, François Sanchez. Coherent soliton pattern formation in a fiber laser. Optics Letters, 2008, 33 (5), pp.524 - 526. 10.1364/OL.33.000524 . hal-03423709

\section{HAL Id: hal-03423709 \\ https://univ-angers.hal.science/hal-03423709}

Submitted on 10 Nov 2021

HAL is a multi-disciplinary open access archive for the deposit and dissemination of scientific research documents, whether they are published or not. The documents may come from teaching and research institutions in France or abroad, or from public or private research centers.
L'archive ouverte pluridisciplinaire HAL, est destinée au dépôt et à la diffusion de documents scientifiques de niveau recherche, publiés ou non, émanant des établissements d'enseignement et de recherche français ou étrangers, des laboratoires publics ou privés. 


\title{
Coherent soliton pattern formation in a fiber laser
}

\author{
Adil Haboucha, Hervé Leblond,* Mohamed Salhi, Andrey Komarov, and François Sanchez \\ Laboratoire POMA, CNRS-FRE 2988, Université d'Angers, 2 Boulevard Lavoisier 49045 Angers Cedex 1, France \\ *Corresponding author: herve.leblond@univ-angers.fr
}

Received November 12, 2007; revised January 11, 2008; accepted January 12, 2008; posted January 22, 2008 (Doc. ID 89665); published February 29, 2008

We report the observation of bound states of 350 pulses in a ring fiber laser mode locked by nonlinear rotation of the polarization. The phenomenon is described theoretically using a multiscale approach to the gain dynamics; the fast evolution of a small excess of gain is responsible for the stabilization of a periodic pattern, while the slow evolution of the mean value of gain explains the finite length of the quasiperiodic soliton train. (c) 2008 Optical Society of America

OCIS codes: $140.3510,190.5530$.

Bound states of two nonconservative solitons have been predicted theoretically in the frame of the complex Ginzburg-Landau (CGL) equation a long time ago [1,2] and were observed in both anomalous [3] and normal [4] dispersion regimes. Three and four pulse solitons have also been predicted $[2,5]$ and observed [6,7]. However, both theoretical predictions and experimental observations are also limited to a few pulses.

On the other hand, indefinitely long periodic (conservative) soliton trains in one dimensional Kerr media have been described theoretically and experimentally. These trains are formed due to the modulational instability of a continuous wave (cw) signal. These are not stable states, since the cw signal is expected to be restored by the so-called FermiPasta-Ulam (FPU) recurrence [8]. An analytical solution of the nonlinear Schrödinger (NLS) equation, which accounts for this phenomenon, has been found [9]. Experiments have been performed for both spatial [10] and temporal [11] solitons. An attempt to stabilize the array leads to the suppression of interactions between solitons [12]. In passive optical resonators, the stabilization of a periodic soliton train has been achieved, yielding a "soliton crystal" $[13,14]$.

In this Letter we report observation of a very long soliton train in a fiber laser, which is stable and phase locked as a bound soliton and periodic as a soliton crystal. However, the soliton train has a finite length, very large with respect to the soliton size, but small with respect to the cavity length $(21 \mathrm{~m}$ or $105 \mathrm{~ns}$ ). This arrangement is similar to a long chain of atoms and can be viewed as a soliton macromolecule.

The setup is presented in Fig. 1. It consists of a unidirectional ring cavity in which the mode locking is achieved thanks to the combined effects of nonlinear polarization rotation and an intracavity polarizer [15]. The total dispersion of the cavity is managed with the use of several fibers with opposite dispersion. The laser operates in the anomalous dispersion regime. We used a double-clad doped fiber with an available output power up to $1 \mathrm{~W}$ when used as a superfluorescent source.

As usual, the orientation of the phase plates allows adjusting the operating regime of the laser. For certain pumping rates and specific orientation of the phase plates, we observe pulse trains of several hundreds of pulses with energy $\sim 15-18 \mathrm{pJ}$ each (Fig. 2). The resolution of the fast detector and of the oscilloscope are not sufficient to observe the detailed structure of the train. Consequently, we observed a periodic square signal without an internal structure; the period is equal to the round-trip time of the cavity. The few oscillations that can be observed at the beginning of the square are not always present and are likely not significant. On the other hand the autocorrelation trace shows a local structure of short pulses repeating periodically (Fig. 3) but cannot show the whole train. In fact, for a set of 100 pulses, the autocorrelation trace has a triangle envelope, but the center undergoes tiny variations, which cannot be detected experimentally because of the noise. The sharpness of peaks in the autocorrelation trace is evidence of the regularity of the periodic pattern. The optical spectrum (Fig. 4) shows a fast modulation, which accounts for the periodic structure of the train and proves the mutual coherence between pulses; they interact and are locked in phase. Recall that for a bunch state, where the pulses are not phase locked, the experimental optical spectrum is not modulated because it results from an averaging over thousands of round trips. The spectrum also presents a global envelope that describes the sech structure of the individual pulse.

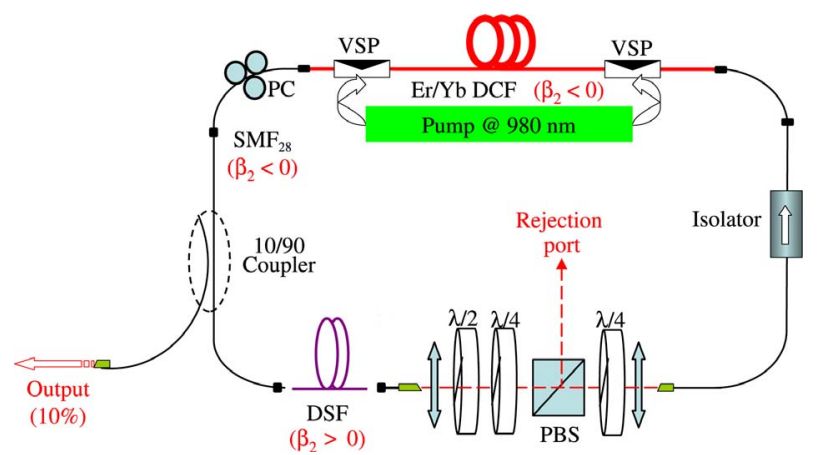

Fig. 1. (Color online) Experimental setup. PBS, polarization beam splitter; DSF, dispersion-shifted fiber $(2.15 \mathrm{~m}$, $\left.\beta_{2}=+0.14 \mathrm{ps}^{2} / \mathrm{m}\right) ; \quad \mathrm{SMF}_{28}, \quad$ standard single-mode fiber $\left(9.6 \mathrm{~m}, \quad \beta_{2}=-0.0217 \mathrm{ps}^{2} / \mathrm{m}\right) ; \mathrm{PC}$, polarization controller; VSP, V-groove side-pumping; Er/Yb DCF, Er/Yb doped double-clad fiber $\left(9 \mathrm{~m}, \beta_{2}=-0.015 \mathrm{ps}^{2} / \mathrm{m}\right)$. 




Fig. 2. (Color online) Oscilloscope trace, the repetition of the pulse train at the fundamental frequency of the cavity. The inset presents the global shape of a train.

The separation between pulses is given by both the modulation of the spectrum and the autocorrelation trace as $\Delta \tau=23 \mathrm{ps}$. From the oscilloscope we get a total length of $8.2 \mathrm{~ns}$, which corresponds to $\sim N=350$ pulses in the train. Using the ansatz

$$
f=\sum_{j=1}^{N} E e^{i(\omega(t-j \Delta \tau)+j \delta \varphi)} \frac{e^{-i C\left[(t-j \Delta \tau)^{2}\right] / 2 \tau^{2}}}{\cosh \left(\frac{t-j \Delta \tau}{\tau}\right)},
$$

including a linear chirp with parameter $C$ and a constant dephasing $\delta \varphi$ between two neighbor pulses, we reconstruct the autocorrelation trace with the above separation and a pulse length $\tau \simeq 900 \mathrm{fs}$. $\delta \varphi$ cannot be accessed experimentally and is set to zero. The optical frequency is $193 \mathrm{THz}$. The simulation is restricted to a 100 pulse train due to limited computer memory. Adjusting the chirp parameter to $C=1.2$ allows to reproduce the experimental width of the spectrum. The agreement in the spectrum (Fig. 4) and the autocorrelation trace (not shown) demonstrates the validity of the above ansatz and estimated parameters.

The observations can be accounted for by using a multiple scale approach [16]. We give herein an outline of the reasoning, to be published in detail elsewhere.

The gain dynamics, in a standard four level model, assuming that the upper and lower nonradiating transitions are very fast, is given by

$$
\frac{\partial g}{\partial t}=-\frac{g}{\tau_{g}}-a g|E|^{2}+\Lambda,
$$

where $g$ is the gain parameter, $E$ the amplitude of the electric field, $\tau_{g}=10 \mathrm{~ms}$ is the relaxation time, $\Lambda$ is the pumping parameter, and $a$ is proportional to the cross section.



Fig. 3. (Color online) Autocorrelation trace showing the periodic local pattern.
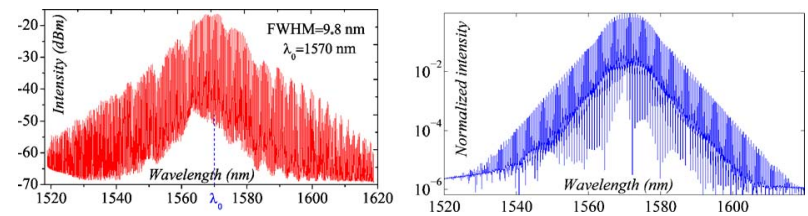

Fig. 4. (Color online) Optical spectrum of the pulse train. Left, experimental; right, reconstructed.

Since $\tau_{g}$ is very large with respect to the duration of the pulse train, and even to the cavity round trip, the relaxation of the gain is slow. However the term proportional to $|E|^{2}$ in Eq. (2) induces fast variations of the gain, at the time scale of the pulse itself. These variations must obviously remain small. Hence the gain suffers small variations approximately a value $g_{0}$, which is constant on a few pulse durations, and evolves slowly according to

$$
\frac{\partial g_{0}}{\partial \tau}=\left(-\frac{1}{\tau_{g}}-a\left\langle|E|^{2}\right\rangle\right) g_{0}+\Lambda .
$$

The fast variations of the gain can be incorporated into the equation evolution for the electric field $E$. The latter, derived as in [17], reads as

$$
\begin{aligned}
i \frac{\partial E}{\partial \zeta}= & i g_{1} E+\left(\frac{\beta_{2}}{2}+i \rho\right) \frac{\partial^{2} E}{\partial T^{2}}+\left(\mathcal{D}_{r}+i \mathcal{D}_{i}\right) E|E|^{2} \\
& -i \mathcal{G} E \int_{-\infty}^{T}\left(|E|^{2}\left(T^{\prime}\right)-\left\langle|E|^{2}\right\rangle\right) \mathrm{d} T^{\prime}
\end{aligned}
$$

where $g_{1}$ is some excess of gain,

$$
\mathcal{G}=g_{0} a \frac{\exp \left(2 g_{0} L\right)-1}{2 g_{0} L},
$$

$L$ being the cavity length. The explicit expression of coefficients $\mathcal{D}_{r}$ and $\mathcal{D}_{i}$ is given in [17]. $\beta_{2}$ $=-0.002 \mathrm{ps}^{2} / \mathrm{m}$ is the group velocity dispersion and $\rho=g_{0} / \omega_{g}^{2}$, where $\omega_{g} / 2 \pi=2.5 \mathrm{ps}^{-1}$ is the spectral gain bandwidth. An equation close to Eq. (4) has been used to describe harmonic mode locking [18].

The formation of a periodic pattern is due to the fast evolution of gain, accounted for by Eq. (4). Indeed, the numerical solution of Eq. (4) shows the existence of a stable steady state that is periodic in time and arises spontaneously, as shown in Fig. 5.



Fig. 5. (Color online) Evolution of the electric field in the laser cavity, starting from a single pulse, spontaneous arising of a periodic regime. 
The pulses are locked in phase, and their separation and intensity are well defined; starting from any initial data in a wide range, the same values of the pulse separation and intensity are obtained when the steady state is reached.

The finite length of the periodic train is not related to the fast evolution of the gain but to the slow one. Each pulse depletes the gain for a certain value $\delta G$, then the initial gain is recovered due to the relaxation and pumping, according to Eq. (3), along the remaining parts of the cavity (Fig. 6).

The depletion $\delta G$ for one pulse can be estimated from the analytical single-pulse solution of Eq. (4) [18] as

$$
\delta G=\mathcal{G}\left|E_{0}\right|^{2} \tau,
$$

where $\tau$ is the pulse duration and $E_{0}$ its peak amplitude. $\tau$ and $E_{0}$ explicitly express in terms of the physical parameters of the laser, the orientation angles of the phase plates especially. Values consistent with the experimental results are obtained for some particular orientation of the latter, which yields $\delta G=22 \times 10^{-6} \mathrm{~m}^{-1}$.

The gain recovered due to pumping and relaxation during the time interval $\Delta T$ between two passes of the soliton train, according to Eq. (3), is

$$
\Delta g=g_{f}-g_{i}=\left(\Lambda \tau_{g}-g_{i}\right)\left(1-\exp \left(-\Delta T / \tau_{g}\right)\right),
$$

$g_{i}$ being the gain immediately after the train and $g_{f}$ being the gain immediately before it at the next round trip. According to the experimental values, $\Delta T \ll \tau_{g}$ and $\Delta g \simeq \Lambda \Delta T$. The number of pulses is hence $N=\Delta g / \delta G \simeq 350$, in accordance with experimental results.

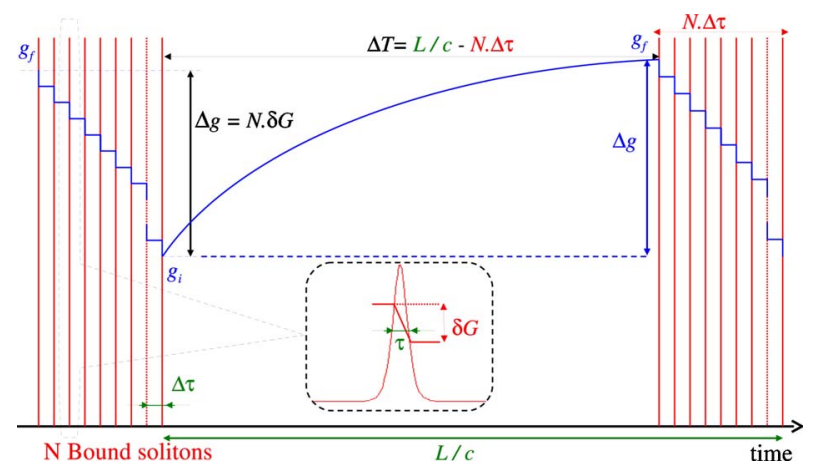

Fig. 6. (Color online) Schema showing the evolution of gain in the cavity, each of the $N$ pulses takes off an amount $\delta G$ of gain, and after the train relaxation restores the amount $\Delta g$.
The finiteness of the wave train is thus explained through a conservation law; the number of pulses in the steady state is such that the decrease of gain due to the amplification of each pulse can be compensated by the pumping.

We observed two time scales in the experiment, corresponding on one hand to the formation of a periodic pattern, which locally compares to a soliton crystal, and on the other hand to the delimitation of a pulse train of definite length. In the same way as an experiment requires different measurement procedures at different scales, the multiscale analysis is allowed to separate them theoretically. Then both small- and large-scale phenomena are accounted for by the theoretical analysis.

\section{References}

1. V. V. Afanasjev, B. A. Malomed, and P. L. Chu, Phys. Rev. E 56, 6020 (1997).

2. N. N. Akhmediev, A. Ankiewicz, and J. M. Soto-Crespo, J. Opt. Soc. Am. B 15, 515 (1998).

3. D. Y. Tang, W. S. Man, H. Y. Tam, and P. D. Drummond, Phys. Rev. A 64, 033814 (2001).

4. A. Hideur, B. Ortaç, T. Chartier, M. Brunel, H. Leblond, and F. Sanchez, Opt. Commun. 225, 71 (2003).

5. H. Leblond, A. Komarov, M. Salhi, A. Haboucha, and F. Sanchez, J. Opt. A 8, 319 (2006).

6. B. Ortaç, A. Hideur, T. Chartier, M. Brunel, Ph. Grelu, H. Leblond, and F. Sanchez, IEEE Photon. Technol. Lett. 16, 1274 (2004).

7. D. Y. Tang, L. M. Zhao, and B. Zhao, Appl. Phys. B 80, 239 (2005)

8. E. Fermi, J. Pasta, and H. C. Ulam, Collected Papers of Enrico Fermi, E. Segrè, ed. (University of Chicago, 1965), Vol. 2, p. 977.

9. N. N. Akhmediev, V. M. Eleonskiĭ, and N. E. Kulagin, Sov. Phys. JETP 62, 894 (1985).

10. C. Cambournac, H. Maillotte, E. Lantz, J. M. Dudley, and M. Chauvet, J. Opt. Soc. Am. B 19, 574 (2002).

11. K. Tai, A. Hasegawa, and A. Tomita, Phys. Rev. Lett. 56, 135 (1986).

12. E. M. Dianov, P. V. Mamyshev, A. M. Prokhorov, and S. V. Chernikov, Opt. Lett. 14, 1008 (1989).

13. S. Rutz and F. Mitschke, J. Opt. B 2, 364366 (2000).

14. B. A. Malomed, A. Schwache, and F. Mitschke, Fiber Integr. Opt. 17, 267 (1998).

15. J. Matsas, T. P. Newton, D. J. Richardson, and D. N. Payne, Electron. Lett. 28, 1391 (1992).

16. H. Leblond, "The reductive perturbation method and some of its applications," J. Phys. B (to be published).

17. H. Leblond, M. Salhi, A. Hideur, T. Chartier, M. Brunel, and F. Sanchez, Phys. Rev. A 65, 063811 (2002).

18. A. Komarov, H. Leblond, and F. Sanchez, Opt. Commun. 267, 162 (2006). 